\title{
Effectiveness of a Matched Filter to Cope with Harsh Phase and Amplitude Modulated EMI
}

\author{
Jonas Lannoo, Tim Claeys, Dries Vanoost, Jonas Van Waes, Student Member, IEEE, \\ Jeroen Boydens, Member, IEEE, and Davy Pissoort, Senior Member, IEEE
}

\begin{abstract}
This paper investigates the effectiveness of a matched filter to cope with continuous wave electromagnetic disturbances that are phase and amplitude modulated from wireless systems, e.g. binary phase shift keying, quadrature amplitude modulation. A wired communication channel between sender and receiver that uses non-return-to-zero-level data encoding is disturbed by those nearby wireless systems. A matched filter is used on the wired communication channel to filter out the additional unwanted wireless disturbance. The bit-error-rate (BER) is calculated after filtering and decoding the received voltages and is used as a metric to compare different kind of disturbances and different levels of sampling frequency. The results show that the matched filter is very effective when the carrier frequency of the disturbance is equal to an integer multiple of the bit frequency, and when not equal to the sampling frequency. This sampling frequency is determined by the bit rate of the desired signal and the oversampling factor on which the matched filter is based. Finally, the filter gain at a bit-error-rate of $0.1 \%$ is determined. This gain shows that an oversampling of 4 times per bit and using a matched filter already results in an average filter gain of 10 to $15 \mathrm{~dB}$.
\end{abstract}

Index Terms-EMI Risk Management, Bit-Error-Rate, Matched Filter, Digital Signal Processing

\section{INTRODUCTION}

In the last decade, a huge digital revolution has happened. Under this revolution a lot of terms are already well known such as Industry 4.0, Internet of Things (IoT), the new $5 \mathrm{G}$ network, retrofitting etc. All these terms have one specific thing in common: wireless communication. Every device has to be able to perform smart operations, be connected to the cloud and be able to interface with other devices. Even systems and locations where safety is of utmost importance like autonomous robots and cars, co-bots and hospitals nowadays comprise a huge set of sensors and wireless electronics that have to work in a safe and safety critical manner. With the contemporary knowledge one would think that this is an easy task, but

This paper is for the Special Section and is an expanded version from the 2019 IEEE International Symposium on EMC and SIPI in New Orleans.

This work is supported by a research grant from the Flemish Agency for Innovation and Entrepreneurship (VLAIO) within the CORNET/TETRA RESSIAR-MID/TransSIMS (HBC.2017.0650) project.

J. Lannoo, D. Vanoost, T. Claeys and D. Pissoort are with the Department of Electrical Engineering, KU Leuven Bruges Campus, Spoorwegstraat 12, 8200 Bruges, Belgium (e-mail: jonas.lannoo@kuleuven.be; dries.vanoost@kuleuven.be; tim.claeys@kuleuven.be; davy.pissoort@kuleuven.be).

J. Boydens is with the Department of Computer Science, KU Leuven Bruges Campus, Spoorwegstraat 12, 8200 Bruges, Belgium (e-mail: jeroen.boydens@kuleuven.be).

Color versions of one or more of the figures in this paper are available online at http://ieeexplore.ieee.org

Manuscript received May xx, 2019 the reality is misleading and disappointing. All these devices have to work seamlessly together in an increasingly polluted ElectroMagnetic (EM) environment, which is indeed not an easy task. Additionally, not only the interference and coupling mechanism (which could be (near/far-) field coupling of electromagnetic waves depending on the distance between victim and source) of innocent wireless devices is important, but also other types of interference such as intentional-electromagnetic interference (IEMI), where it is the goal of disturbing a system intentionally. This is often done in malicious practices or for warfare purposes where hundreds or thousands of volts per meter are used to disrupt a system. The approach of adding a matched filter at the receiver of susceptible communication channels could improve the BER significantly, keeping in mind that other hardware parts are necessary to protect the physical hardware (gates/transistors/junctions/capacitors) [1].

To create a system that is able to work perfectly in an EM environment and that can perform safety critical operations, the fields of Electromagnetic Compatibility (EMC) and Functional Safety (FS) have to be combined. In the literature this has resulted in the discipline of ElectroMagnetic Interference (EMI) Risk Management [2], [3], [4]. This EMI Risk Management uses specific risk analysis [5] and techniques and measures (T\&M's) to achieve EMI Resilience [6] and is extensively described in an IET Code of Practice [7] and in Project IEEE P1848 [8]. The T\&M's that are used are split in to two major sections, hardware based techniques and software based techniques. The latter comprises of methods to detect and/or correct fault in the safety critical systems. This can be achieved by using Error Detection Codes (EDCs) and Error Correction Codes (ECCs) and is already used to create EMI Resilience [9], [10]. The hardware based techniques consist of methods to improve the performance of communication systems by introducing inversion [11], spatial [12], [13] and time [14] diversity. Another method to improve the communication is by adding a matched filter to a communication system [15].

It is well known that a matched filter performs well for stochastic or random noise such as Additive White Gaussian Noise (AWGN) and that it is commonly used in both wired and wireless systems. In wireless systems different types of matched filters are often used to correlate the incoming waveforms with their matching signature waveforms [16] or as a final step in a wireless receiver to down-sample and remove inter-symbol-interference (ISI) and passband ripple [17]. Furthermore, in both systems it is often used to reduce the BER for a certain Signal-to-Noise Ratio (SNR) in normal channels 
or even Rayleigh fading multipath channels [18]. In [19], the performance of a matched filter was investigated when exposed to a harsh continuous wave disturbance. The results showed that a matched filter is most effective when the frequency of the continuous wave $(\mathrm{CW})$ disturbance is an integer multiple of the sampling frequency or when the frequency of the $\mathrm{CW}$ disturbance is significantly larger than the frequency of the transmitted signal. Of course, the EM environment does not only consists of $\mathrm{CW}$ disturbances. As already stated, most of the EM environment consist of intentional wireless communications. In this paper, the effectiveness of a matched filter is tested against four types of digital wireless communications, mostly used in current protocols.

This paper is organised as follows. In Section II the theoretical background of a matched filter is briefly explained. Section III gives an overview of the construction of the wired and wireless communication signals and their properties. Section IV describes the simulation method and calculation of the Bit-Error-Rate (BER) and Filter Gain (FG). Section V shows the simulation results and elaborates on the effectiveness of the matched filter when coping with the phase and amplitude modulated disturbance signals. Finally, Section VI draws concluding remarks.

\section{THE MATCHED FILTER}

In digital communication systems and digital signal processing, a matched filter is often used to maximise the SNR of the receiving signal. Doing so, it minimizes the BER to get the maximum performance of the communication system. The matched filter makes use of the property that the incoming noise is random or stochastic and that it has an average time domain value that is equal to zero. A common example is AWGN, which represents thermal noise as can be found in almost any component or system. The implementation of such a matched filter can be done in several ways.

The first implementation method is by using the matched filter as a linear filter, where the filter uses the signature (or waveform) $s_{1}$ of the required signal (in this case the waveform of a digital '1'). The impulse response or transfer function $h$ of the filter is found by inverting the signature in time and performing a complex conjugate (indicated with $*$ ) on $s_{1}$.

$$
h[m]=s_{1}[T-m]^{*}
$$

In (1), $m$ indicates the index of the sample in one bit. In this paper, the number of samples per bit is 1 (no matched filter), 4 or 8 . The $\mathrm{M}$ samples are uniformly distributed over the bit period, e.g. for $\mathrm{M}=4$ the bit is sampled at $t=\left[1 / 4 T_{\mathrm{BIT}} ; 1 / 2 T_{\mathrm{BIT}} ; 3 / 4 T_{\mathrm{BIT}} ; T_{\mathrm{BIT}}\right]$. In Linear Time Invariant systems like this, the filter is applied by performing a convolution of the transfer function $h$ with the received signal $x$ :

$$
y[k]=\sum_{m=0}^{M-1} h[k-m] x[m]=(h * x)[k] .
$$

In (2), $x$ exists out of the superposition of the desired encoded signal and the phase and amplitude shift keying disturbance. Because of this superposition, distributivity can be applied

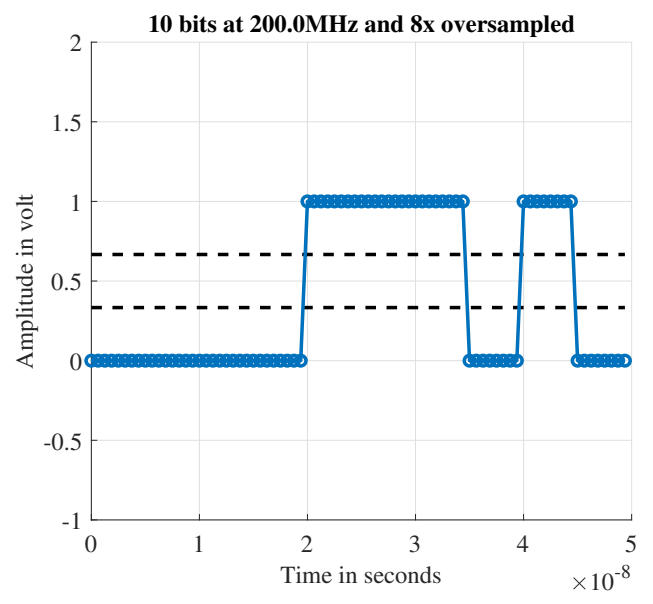

Fig. 1. NRZ-L encoding of 10 bits ('0000111010'), transmitted at $200 \mathrm{MHz}$ and oversampled $M=8$ times. The black dashed lines represent the decoding thresholds.

resulting in two separate convolutions with the transfer function of the filter. Once with the desired signal and once with solely the disturbance. The convolution with the desired signal results in the exact bit stream, while the convolution with the disturbance calculates in essence the average of the sampled disturbance. Afterwards both are added up. This explains why a matched filter is so effective for Gaussian noise, indeed because the average is almost zero.

The second method of implementing a matched filter uses the same reasoning, but is done by only adding and multiplying the received samples at the end of every received bit. This results in an easier implementation in programmable hardware, because it only uses addition and multiplication blocks. The received disturbed sample values in one bit period are multiplied with the signature of the matched filter, added up and multiplied with a factor, to average the outcome. Again, the sum of the samples results in a minimisation of the stochastic or random noise because the average of those types of noise is near-zero. This method is also known as the matched correlator, the received signal is correlated with the signature of an encoded '1' [15], [19].

$$
y\left[T_{\mathrm{BIT}}\right]=\frac{1}{T_{\mathrm{BIT}}} \sum_{m=0}^{M-1} s_{1}[m] x[m]
$$

The last method is equal to the first method, but instead of applying the linear filter in the time domain, it is now used as frequency domain filter. Therefore the Discrete Fourier Transform (DFT) of $s_{1}$ is taken and multiplied with the DFT of the received signal. An extra inverse DFT is used to convert back to the time domain and sampled at the bit frequency, and decode those values to get the received bits. In this paper the first method (convolution in time domain) is used at all times to calculate the received values after the matched filter.

\section{Communication Channel Setup}

The communication between the sender and receiver which is interfered by the phase and amplitude modulated disturbance can be split up in two parts. The first part comprises of 


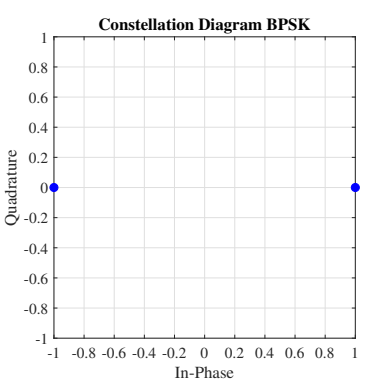

(a) BPSK

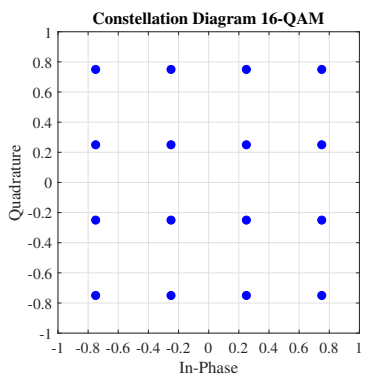

(c) 16-QAM

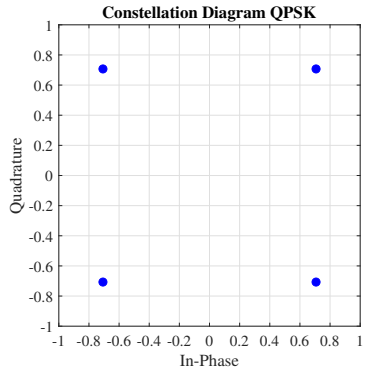

(b) QPSK

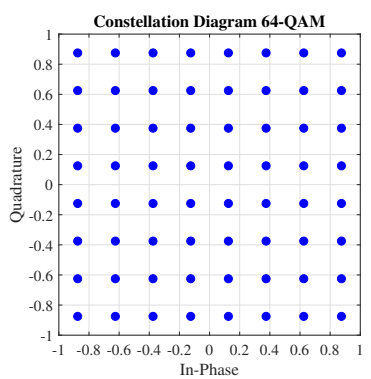

(d) 64-QAM
Fig. 2. The constellation diagrams of the four phase and amplitude shift keying disturbances.

the creation of the wired communication signal, which is the desired encoded bit stream. The wired communication channel can be a micro-strip on a PCB or a wire, but is in this case an ideal transmission line between transmitter and receiver is used. The second part involves the creation of a wireless communication channel that interferes with the wired communication. Both parts have their specific parameters which are discussed in the following subsections.

\section{A. Desired Encoded Bit Stream}

The wired communication between sender and receiver uses Non-Return-to-Zero Level (NRZ-L) encoding, which means that a digital ' 0 ' is translated to $0 \mathrm{~V}$ and a digital ' 1 ' to a high voltage, in this case $1 \mathrm{~V}$. A set of $N$ (e.g. $N=100$ ) random bits are transmitted at a speed defined as $f_{\mathrm{BIT}}$, while a bit is received every bit-period $T_{\mathrm{BIT}}$. The bits are oversampled $M$ times, resulting in a sampling frequency of $f_{s}=M f_{\mathrm{BIT}}$. An example of 10 bits transmitted over the communication channel is shown in Fig. 1. The oversampling factor $M$ decides how many samples the matched filter uses to apply the digital filtering. More precisely, the matched filter will multiply and accumulate the $M$ sampled values in one bit period with the impulse response of the filter as shown in (4),

$$
v_{\text {BIT }}[n]=\frac{1}{M} \sum_{m=0}^{M-1} h[m] x[m]
$$

concluding in the received value for that bit. The receiver performs a decoding of the bit using two thresholds. The threshold to determine a digital ' 1 ' is $2 / 3$ of $1 \mathrm{~V}$, while the threshold for a digital ' 0 ' is $1 / 3$ of $1 \mathrm{~V}$. When the received voltage is located between the thresholds, the bit is concluded as faulty in our study, to create a worst-case BER.
TABLE I

SUMMARY OF BASEBAND SIGNAL VALUES

\begin{tabular}{|l|c|c|c|}
\hline Signal & RMS Value & Peak Value & PAPR \\
\hline$V_{\text {BIT }}$ & $\sqrt{2} / 2 \mathrm{~V}$ & $1 \mathrm{~V}$ & $3.01 \mathrm{~dB}$ \\
\hline$V_{\text {CW }}$ & $1 \mathrm{~V}$ & $1 \mathrm{~V}$ & $0 \mathrm{~dB}$ \\
\hline$V_{\text {BPSK }}$ & $1 \mathrm{~V}$ & $1 \mathrm{~V}$ & $0 \mathrm{~dB}$ \\
\hline$V_{\text {QPSK }}$ & $1 \mathrm{~V}$ & $1 \mathrm{~V}$ & $0 \mathrm{~dB}$ \\
\hline$V_{16-\mathrm{QAM}}$ & $\sqrt{10} / 4 \mathrm{~V}$ & $3 \sqrt{2} / 4 \mathrm{~V}$ & $2.55 \mathrm{~dB}$ \\
\hline$V_{64-\mathrm{QAM}}$ & $\sqrt{42} / 8 \mathrm{~V}$ & $7 \sqrt{2} / 8 \mathrm{~V}$ & $3.68 \mathrm{~dB}$ \\
\hline
\end{tabular}

\section{B. Phase and Amplitude Modulated Disturbance}

As the authors stated in the introduction, several harsh EM fields and disturbances can be used to (un-)intentionally interfere with a system. The disturbances that are considered in this paper are: Binary Phase Shift Keying (BPSK), Quadrature Phase Shift Keying (QPSK), 16-bit Quadrature Amplitude Modulation (16-QAM) and 64-bit Quadrature Amplitude Modulation (64-QAM). The comparison with unmodulated Continuous Wave $(\mathrm{CW})$ disturbance is included in this paper to investigate the effect of the phase and/or amplitude shifts on to the performance of the matched filter. The constellation diagrams of the phase and amplitude modulated signals summarize the possible set of symbols for each modulation type and are shown in Fig. 2. The baseband values are the values that are shown in the constellation diagrams and are used to calculate the RMS value,

$$
V_{\mathrm{RMS}}=\sqrt{\frac{1}{S} \sum_{s=0}^{S-1}\left|x_{s}\right|^{2}},
$$

where $S$ indicates how many symbols are possible for each modulation type (e.g. $S=2$ for BPSK, $S=4$ for QPSK). Equation (5) can be similarly used for the other disturbance types. Furthermore, the symbols are used to calculate the peak value,

$$
V_{\text {peak }}=\max \left(\left|x_{s}\right|\right) \quad \text { with } s=0,1, . . S-1
$$

and the Crest Factor or Peak to Average Power (PAPR) ratio in Decibel $(\mathrm{dB})$ :

$$
\text { PAPR }_{\mathrm{dB}}=20 \log _{10}\left(\frac{\left|V_{\text {peak }}\right|}{V_{\mathrm{RMS}}}\right) .
$$

To shift the baseband symbols to the passband, which is the disturbance that will be superposed with the bit signal, the symbols are multiplied with a carrier, having a frequency $f_{c}$. The in-phase (I) and quadrature (Q) component of the symbol, which are the real and imaginary components respectively, are multiplied with a cosine and sine version of the carrier, and summed in (8).

$$
\begin{aligned}
V_{s}= & A_{c} I_{s} \cos \left(2 \pi f_{c}(t-\Delta t)\right) \\
& +A_{c} Q_{s} \sin \left(2 \pi f_{c}(t-\Delta t)\right)
\end{aligned}
$$

Where $\Delta t$ represents the moment in time when the disturbance hits the communication channel, which is random and uniformly distributed. Further, $f_{c}$ is the frequency of the carrier and $A_{c}$ is the amplitude of the carrier. One more important 


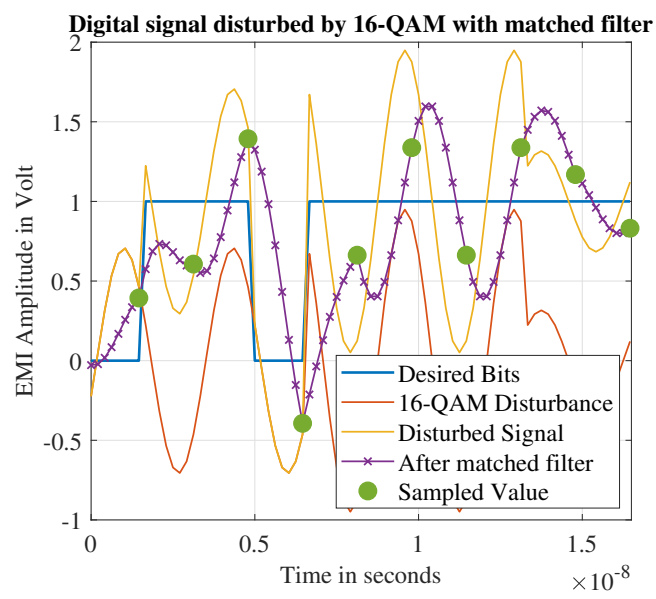

Fig. 3. Example of 10 bits ('0110111111') NRZ-L encoded disturbed by a 16-QAM signal. The wireless disturbance is superposed with the wired transmitted signal to create the disturbed signal. The matched filter is applied to the receiving samples and the output samples at the bit periods are concluded. The resulting bits are 'xx10x1x111' where ' $\mathrm{x}$ ' denotes a faulty bit, resulting in a BER of $40 \%$.

factor is the symbol rate $f_{\mathrm{SYM}}$ of the phase modulation method. In wireless communication the symbol rate is determined by the bit rate and the modulation type, i.e. the number of bits fitting in one symbol (1 for BPSK, 2 for QPSK, 4 for 16QAM and 8 for 64-QAM). To avoid confusion, the bit rate of the disturbance is not used in this paper, instead only the symbol rate is defined. The symbol rate defines the bandwidth $\left(\mathrm{BW}=2 f_{\mathrm{SYM}}\right)$ of the disturbance signal that is transmitted by a wireless communication system. The number of symbols that are simulated is a random set of 100 times the amount of symbols that exist for that disturbance type. Additionally, the whole disturbance is swept over all the bits in steps of 1/100th of the symbol length. For example when using 64-QAM as a disturbance this will result in 64000000 bit evaluations.

\section{Calculation of the Bit-ERror-Rate And Filter GAIN}

The overall BER is considered to be the metric to evaluate the effectiveness of the matched filter. The construction of a disturbed bit stream is shown in Fig. 3, which shows the transmitted bits, the 16-QAM disturbance, the superposition of the latter two being the disturbed signal, the values after the matched filter calculated using (2) and the sampled values after using (3). The sampled values are then decoded to bits as described in Section III-A and are compared to the original transmitted bits to conclude a BER. The BER is calculated in function of a Signal-to-Noise Ratio (SNR), which is determined by the ratio of the power of the desired signal to the power of the disturbance signal. The RMS values are used for both signals and the SNR is calculated in the following way:

$$
\mathrm{SNR}=20 \log _{10}\left(\frac{V_{\mathrm{BIT}, \mathrm{RMS}}}{V_{\mathrm{EMI}, \mathrm{RMS}}}\right)
$$

The RMS values of the desired signal and all considered disturbances are summarised in Table I. Note that the RMS values of the disturbances are calculated using the possible symbol values, in the baseband. When calculating the RMS value in the passband, all RMS values have to be multiplied by $\sqrt{2} / 2$ because of the multiplication with the carrier. The Peak to Average Power Ratio (PAPR) is calculated here because it has an influence on the noise strength that is necessary to start creating bit errors. The PAPR tells us the ratio of the peak power in respect to the RMS power of the wireless signal in $\mathrm{dB}$. The peak value is equal to the maximum amplitude of the carrier. When this maximum amplitude is higher than the lowest threshold that is used to decode the desired signal, a possible bit error can occur.

Furthermore, a Filter Gain (FG) is defined at a BER equal to $0.1 \%$. This parameter represents how much SNR is gained by applying a matched filter to achieve an equal BER. The authors assume that the remaining $0.1 \%$ of bit errors that occur are covered by other methods (e.g. Error Detection Codes (EDC) or Error Correction Codes (ECC)). FG is also used as the amount of power that you can decrease your wired transmitting amplifier with, to achieve the same BER.

\section{Simulation Results}

First, a baseline is simulated where a bit stream is disturbed by all phase and amplitude modulation types without a matched filter. Next, the same situations are simulated using a matched filter. And finally, the effect of changing the possible parameters of the desired signal and the harsh disturbance are investigated.

\section{A. Baseline Simulation Without Matched Filter}

As a baseline a set of 100 bits is disturbed multiple times with all five types of the considered phase and amplitude modulated disturbances separately. When using no matched filter and calculating the bit error rate in function of the SNR, the reference BER-curves are shown in Fig. 4.

The reference situation in Fig. 4 shows almost equal behaviour for all five types of disturbance. However, there is a distinction between the PSK methods and the QAM methods due to the different possible amplitudes of the baseband symbols. The BER is slightly different because the QAM modulation schemes involve symbols that have a norm (or length) that is smaller than the peak value, where for PSK methods the norm of the symbol vectors or the amplitude of the modulated carrier is equal at any point in time. Moreover, when looking closely to the SNR value where the bit errors start to occur, four specific properties can be recognised in Fig. 4:

A) The SNR where the BER starts for CW, BPSK and QPSK is equal to $6.532 \mathrm{~dB}$, which is the corresponding value for the threshold of $1 / 3$ or $2 / 3$ to detect a digital ' 0 ' or ' 1 '. When the amplitude of the disturbance rises above or falls below this value due to the superposed disturbance, possible bit errors start to occur.

B) For CW, BPSK and QPSK there is a change in BER at an SNR of $0.512 \mathrm{~dB}$, which is equivalent to an amplitude of $0.667 \mathrm{~V}$ or $2 / 3$. This indicates that more bit errors start 


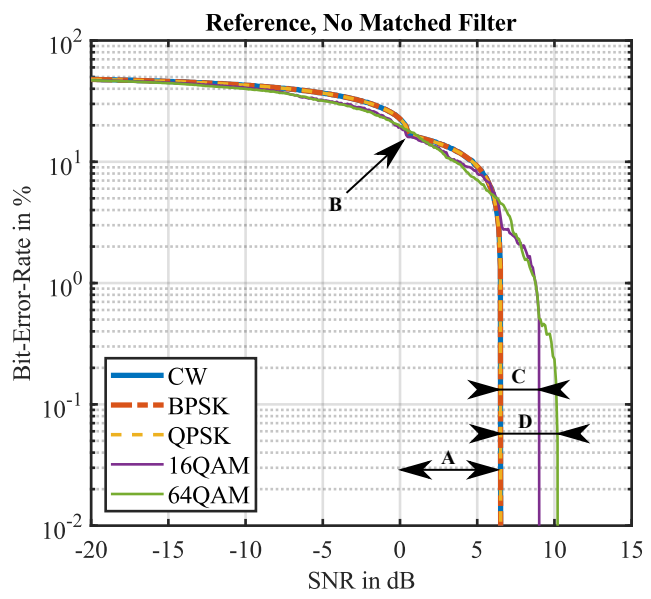

Fig. 4. BER in function of SNR when using no matched filter, at a bit rate $f_{\mathrm{BIT}}=200 \mathrm{MHz}$, an EMI carrier $f_{c}=500 \mathrm{MHz}$ and a symbol rate $f_{\mathrm{SYM}}=1 \mathrm{MHz}$.

to occur when the amplitude of the disturbance is high enough to cross both thresholds.

C) The SNR where bit errors start to occur for 16-QAM is equal to $9.08 \mathrm{~dB}$, which corresponds with an increase of $2.55 \mathrm{~dB}$ in comparison with the SNR where the BER starts of $\mathrm{CW}$. This difference is equal to the PAPR of 16-QAM.

D) The SNR where bit errors start to occur for 64-QAM is equal to $10.21 \mathrm{~dB}$, which corresponds with an increase of $3.68 \mathrm{~dB}$ in comparison with the SNR where the BER of of $\mathrm{CW}$ starts. This difference is equal to the PAPR of 64-QAM.

Furthermore, changing the bit frequency of the desired bit stream or changing the frequency of the EMI carrier does not change the BER in function of the SNR, and this for all types of disturbances. The BER does not change because all possible values of the disturbance are considered, so when sampling once only the amplitude of the disturbance decides if bit errors

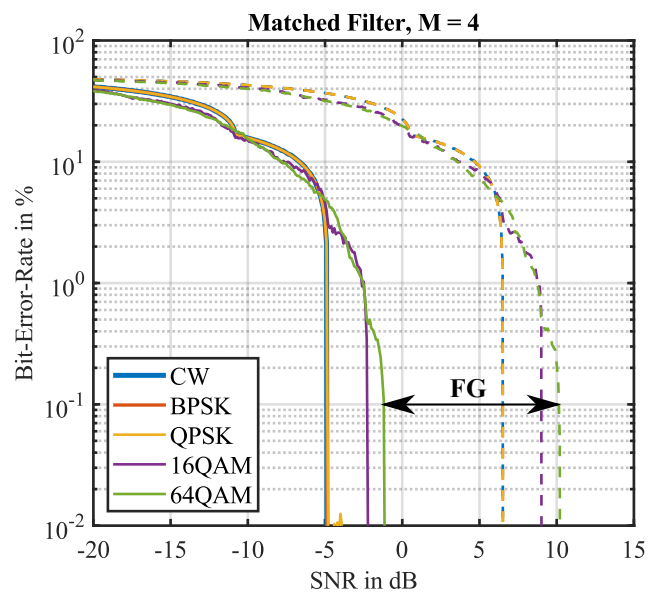

Fig. 5. Definition of the filter gain (FG) for all disturbances when using a matched filter with $M=4$ at n $f_{c}=500 \mathrm{MHz}$, an $f_{\mathrm{BIT}}=200 \mathrm{MHz}$. The coloured dashed lines represent the BER without using a matched filter for the corresponding disturbance.

occur. This effect is shown in Fig. 6 for BPSK and 16-QAM, and is similar for the other disturbances.

\section{B. Simulation With Matched Filter}

First, the effect of a matched filter that uses 4 samples per bit is considered.The difference compared to the reference in Fig. 4 is shown in Fig. 5. The change in BER in function of $f_{c}$ and the SNR is shown in Fig. 7 for BPSK and 16-QAM. The frequency of the wired signal is $200 \mathrm{MHz}$, which results, in combination with 4 samples per bit, in a sampling frequency $f_{s}$ of $800 \mathrm{MHz}$. When analysing Fig. 7 two properties are found in function of $f_{\mathrm{BIT}}$ and $f_{s}$. On the one hand, after a matched filter with 4 samples per bit, the integer multiples of the bit frequency $f_{\text {BIT }}$ show up as improvements of the BER in comparison with the reference in Fig. 6. On the other hand, the integer multiples of the sampling rate $f_{s}$ show up as totally no improvement of the BER. In the latter case, the samples that are taken of the disturbance are 4 times the same sample,

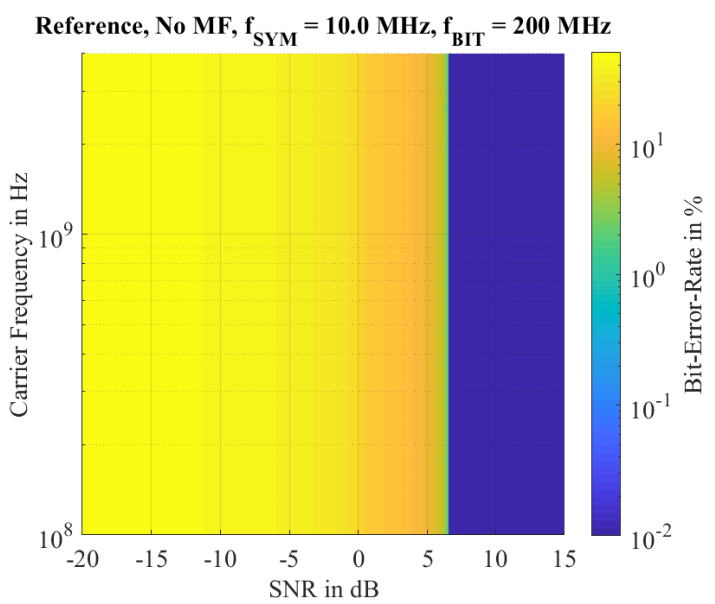

(a) BPSK

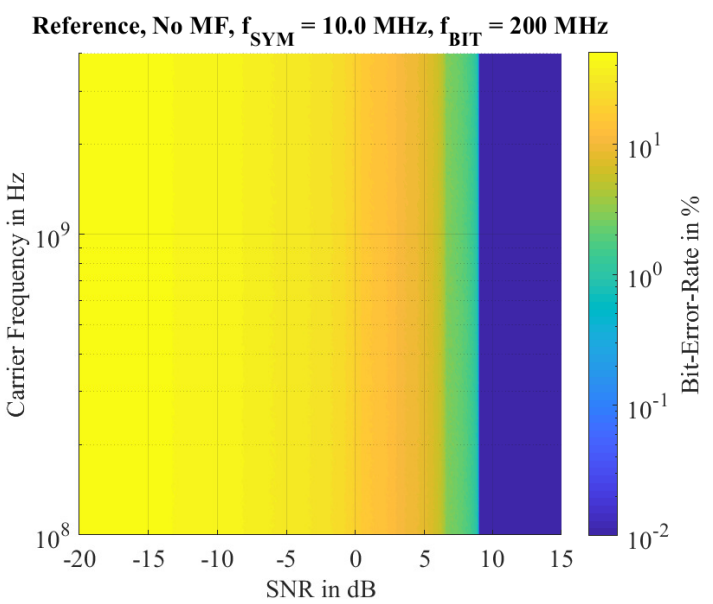

(b) 16-QAM

Fig. 6. The BER for BPSK and 16-QAM in function of $f_{c}$ for an $f_{\mathrm{SYM}}=10 \mathrm{MHz}$, an $f_{\mathrm{BIT}}=200 \mathrm{MHz}$ and no matched filter. 


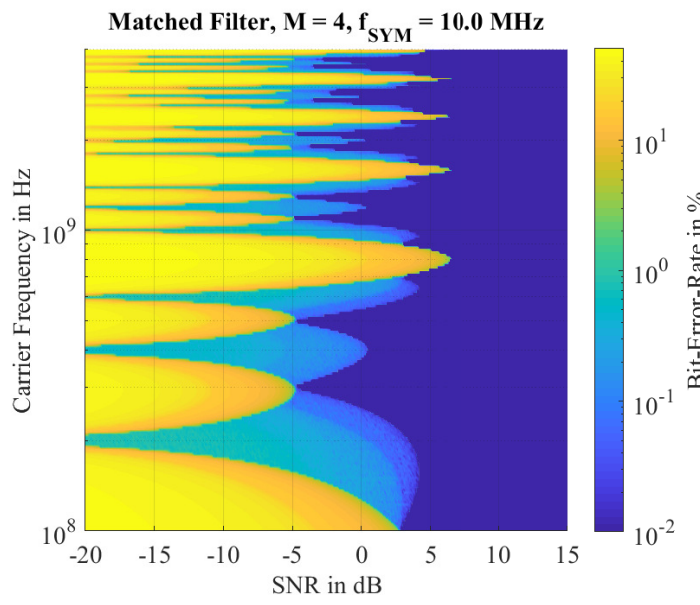

(a) BPSK

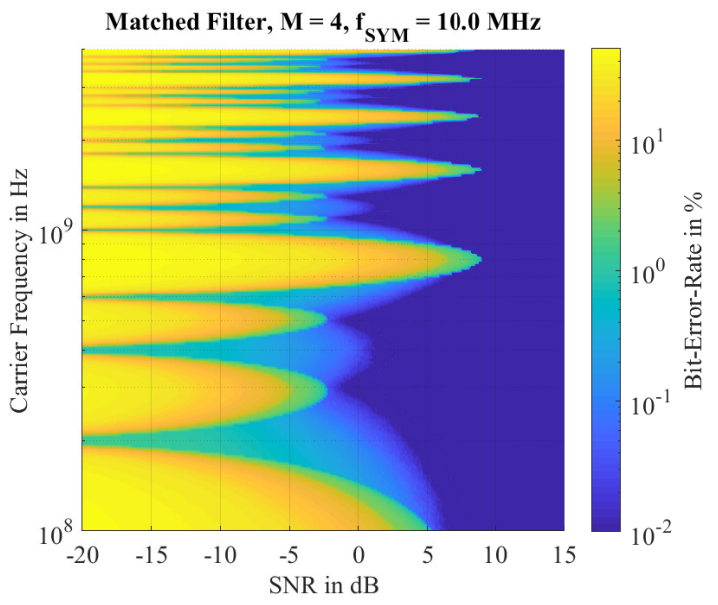

(b) 16-QAM

Fig. 7. The BER for BPSK and 16-QAM in function of $f_{c}$ for an $f_{\mathrm{SYM}}=10 \mathrm{MHz}$, an $f_{\mathrm{BIT}}=200 \mathrm{MHz}$ and $M=4$.

and thus taking an average results in a disturbance that has been sampled only once per receiving bit. Furthermore, the changes in BER due to the decoding thresholds or the PAPR are very visible in Fig. 7 as sudden colour changes.

The symbol rate $f_{\mathrm{SYM}}$ in Fig. 7 is equal to $10 \mathrm{MHz}$ and is fixed for every $f_{c}$. As already mentioned in Section III-B, the symbol rate determines the number of carrier periods in one symbol, and thus how many carrier periods have to be observed before a possible phase and/or amplitude shift occurs. This change in phase and/or amplitude results in the matched filter to not filter out the disturbance because the average of the sampled points has a smaller change of being near-zero. The influence of the symbol rate on the BER for QPSK is investigated and shown in Fig. 8. The reference BER in function of the SNR without matched filter is shown in contrast with the results with matched filter for three different symbol rates. The increase in the symbol rate shows that the matched filter is indeed having more difficulties to filter out the disturbance signal. Note that A) and B) in Fig. 8 show that an increase of the symbol rate by a factor of 10 increases the chance of a bit error for the same SNR also by a factor of 10 . The effect of increasing $f_{\mathrm{SYM}}$ is equal for all considered disturbances in this paper and is also recognizable by analysing the BER lower limit in Fig. 9 and 10.

Taking a cross-section of Fig. 7 at an SNR $-5 \mathrm{~dB}$ results in Fig. 9, which shows the response of the BER in function of the EMI carrier frequency for all types of disturbances. Increasing the number of samples per bit to $M=8$, meaning that matched filtering is applied on 8 samples per bit, the BER is decreased even more and is shown in Fig. 10.

To have an idea of how effective the matched filter is, a specific BER value is chosen, and the required SNR to reach that number of bit errors is determined. That specific BER is determined by the system that is connected to the receiver. The receiver could have extra safety measures to solve the extra bit errors that the matched filter cannot filter out. In the case of this paper, the chosen BER is equal to $0.1 \%$ which means that 1 in 1000 bits can be wrong. The effectiveness of the filter is

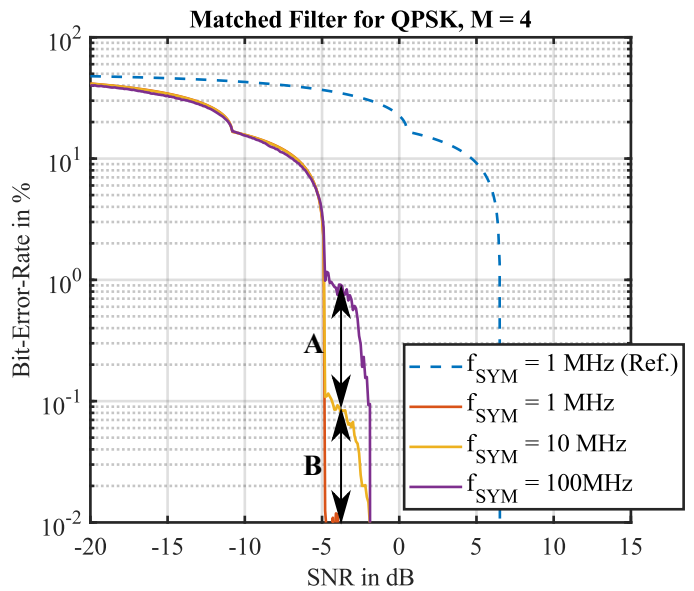

Fig. 8. The BER for QPSK in function of the SNR for different values of the symbol rate $f_{\mathrm{SYM}}$ at an $f_{c}=500 \mathrm{MHz}$, an $f_{\mathrm{BIT}}=200 \mathrm{MHz}$ and $M=$ 4.

tested with and without matched filter for different number of samples. The (positive) difference of SNR that is required to gain the same BER is called the Filter Gain (FG). This means that with the use of a matched filter, less transmission power can be used, to achieve the same BER. The FG for the case of no matched filter versus a matched filter with 4 sample points is defined in Fig. 5.

To determine the effectiveness of a matched filter, the term Filter Gain (FG) has been defined. The FG, in $\mathrm{dB}$, is the difference in SNR between a matched filter and no filter for a specific BER value. This specific BER value is determined by the application of the wired communication. To further decrease the bit error, ECCs can be applied. The effectiveness of those codes is specified by the term Coding Gain (CG), but is not considered in this paper. The BER where the FG is determined is specified on a BER equal to $0.1 \%$. Meaning that 1 in 1000 bits can be wrong. The FG for a matched filter with 4 sample points is shown in Fig. 5.

The FG for a CW, BPSK, QPSK and 16-QAM disturbance 


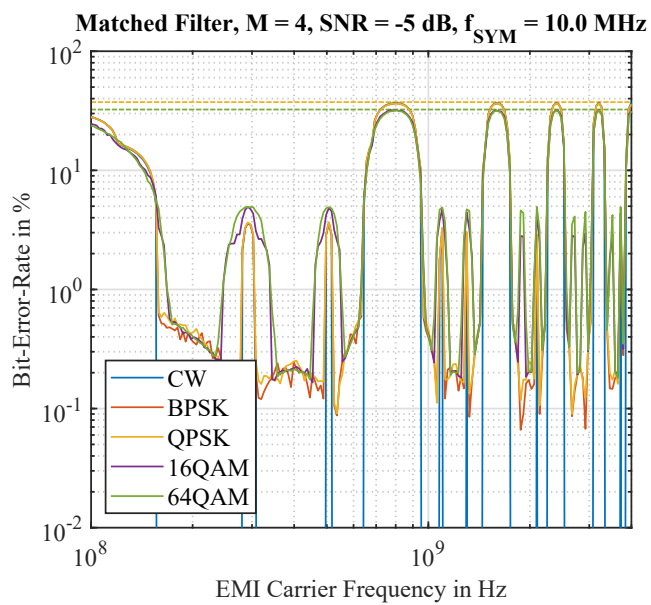

Fig. 9. The BER for all disturbances at an SNR of $-5 \mathrm{~dB}$ in function of the carrier frequency $f_{c}$ of the disturbance at an $f_{\mathrm{SYM}}=10 \mathrm{MHz}$, an $f_{\mathrm{BIT}}=$ $200 \mathrm{MHz}$ and $M=4$. The sample frequency $f_{s}$ is equal to $800 \mathrm{MHz}$. The reference value of the BER at $-5 \mathrm{~dB}$ for each of the disturbances without applying a matched filter is shown with dashed lines.

and is shown in Fig. 11. Fig. 11a shows the FG in function of the EMI CW carrier frequency $f_{c}$ and shows that there is a gain of at least $10 \mathrm{~dB}$ when not having a $\mathrm{CW}$ carrier frequency which is an integer multiple of the sampling frequency $f_{s}$. Furthermore, Fig. 11b, 11c and 11d have an FG that is similar to the $\mathrm{FG}$ of the $\mathrm{CW}$ disturbance, when the symbol rate $f_{\mathrm{SYM}}$ is below a certain frequency.

Finally, increasing the number of samples per bit again to 8 , the FG is further increased to $15 \mathrm{~dB}$. This result is shown in Fig. 12 for a CW and QPSK disturbance.

\section{CONCLUSION}

In this paper a wired communication channel that is NRZ-L encoded is disturbed by a wireless communication channel with phase and amplitude shift keying properties. To filter out the unwanted disturbances, a matched filter is created using the signature of an encoded ' 1 '. Furthermore, five types of disturbances are considered that are superposed on to the desired signal, CW, BPSK, QPSK, 16-QAM and 64-QAM. When using no matched filter the BER baseline is defined and the effect of using different modulation schemes is shown. When introducing a matched filter with a finite number of samples per bit, it is shown that the matched filter is very effective at integer multiples of the desired bit frequency, excluding the integer multiples of the sampling frequency. When the EMI symbol rate is small enough, there is no big of a difference between a $\mathrm{CW}$ disturbance and a phase and amplitude modulated disturbance. When introducing a higher symbol rate than the carrier frequency, multiple phase changes in one period of the EMI carrier wave are introduced. The matched filter has more difficulties filtering out the disturbance and the performance is decreased. This paper shows that using a matched filter can be a very effective way of filtering out unwanted disturbances at certain frequencies, and that maximising the sampling rate improves the maximum possible disturbance frequencies that can be filtered out. The results show that a matched filter with 4 sample points per receiving

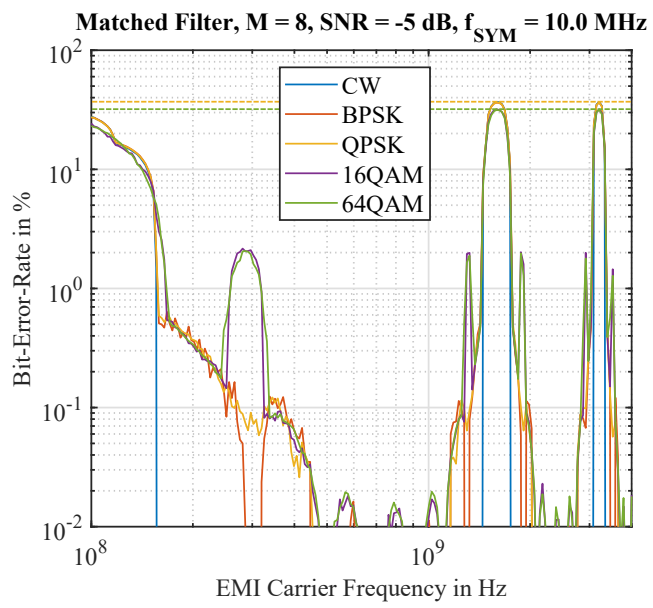

Fig. 10. The BER for all disturbances at an SNR of $-5 \mathrm{~dB}$ in function of the carrier frequency $f_{c}$ of the disturbance at an $f_{\mathrm{SYM}}=10 \mathrm{MHz}$, an $f_{\mathrm{BIT}}=$ $200 \mathrm{MHz}$ and $M=8$. The sample frequency $f_{s}$ is equal to $1.6 \mathrm{GHz}$. The reference value of the BER at $-5 \mathrm{~dB}$ for each of the disturbances without applying a matched filter is shown with dashed lines.

bit already provides a filter gain around $10 \mathrm{~dB}$, which can be further improved by increasing the number of samples per bit.

\section{REFERENCES}

[1] W. A. Radasky, C. E. Baum, and M. W. Wik, "Introduction to the special issue on high-power electromagnetics (HPEM) and intentional electromagnetic interference (IEMI)," IEEE Transactions on Electromagnetic Compatibility, vol. 46, no. 3, pp. 314-321, 2004.

[2] D. Pissoort and K. Armstrong, "Why is the IEEE developing a standard on managing risks due to EM disturbances?" in 2016 IEEE International Symposium on Electromagnetic Compatibility (EMC), July 2016, pp. 7883.

[3] F. Sabath, "A systematic approach for electromagnetic interference risk management," IEEE Electromagnetic Compatibility Magazine, vol. 6 , no. 4, pp. 99-106, 2017.

[4] K. Armstrong, "Techniques and measures to manage functional safety and other risks with regard to electromagnetic disturbances," in 2018 IEEE 4th Global Electromagnetic Compatibility Conference (GEMCCON), 2018, pp. 1-5.

[5] E. Genender, H. Garbe, and F. Sabath, "Probabilistic risk analysis technique of intentional electromagnetic interference at system level," IEEE Transactions on Electromagnetic Compatibility, vol. 56, no. 1, pp. 200-207, 2014.

[6] D. Pissoort, J. Lannoo, J. V. Waes, A. Degraeve, and J. Boydens, "Techniques and measures to achieve EMI resilience in mission- or safety-critical systems," IEEE Electromagnetic Compatibility Magazine, vol. 6, no. 4, pp. 107-114, Fourth 2017.

[7] The IET, Code of Practice for Electromagnetic Resilience. Institution of Engineering \& Technology, 2017. [Online]. Available: https://books.google.be/books?id=uN4FkAEACAAJ

[8] K. Armstrong, A. Duffy, "Techniques \& measures to manage functional safety and other risks with regard to electromagnetic disturbances," International Organization for Standardization, IEEE Standard P1848, 2017.

[9] J. Van Waes, J. Lannoo, J. Vankeirsbilck, A. Degraeve, J. Peuteman, D. Vanoost, D. Pissoort, and J. Boydens, "Effectiveness of hamming single error correction codes under harsh electromagnetic disturbances," in 2018 International Symposium on Electromagnetic Compatibility (EMC EUROPE), Aug 2018, pp. 271-276.

[10] J. Van Waes, D. Vanoost, J. Vankeirsbilck, J. Lannoo, D. Pissoort, and J. Boydens, "Resilience of error correction codes against harsh electromagnetic disturbances: Fault mechanisms," IEEE Transactions on Electromagnetic Compatibility, pp. 1-11, 2019. 


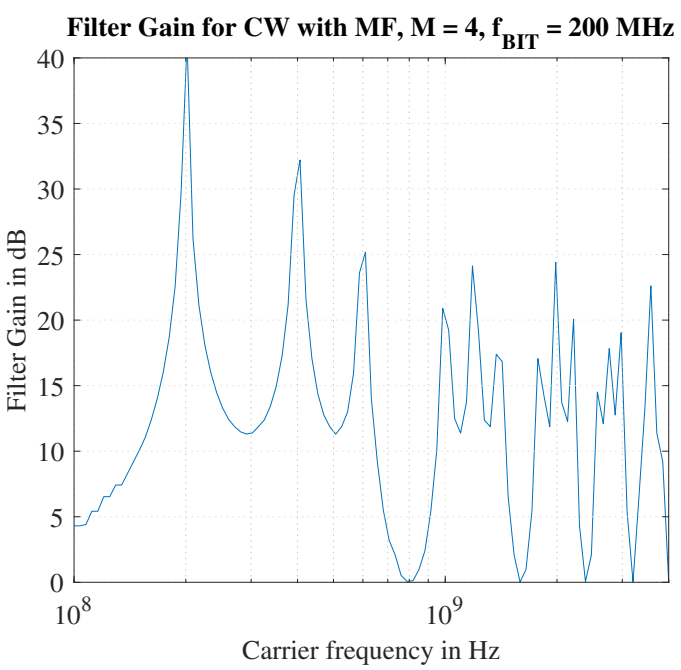

(a) $\mathrm{CW}$

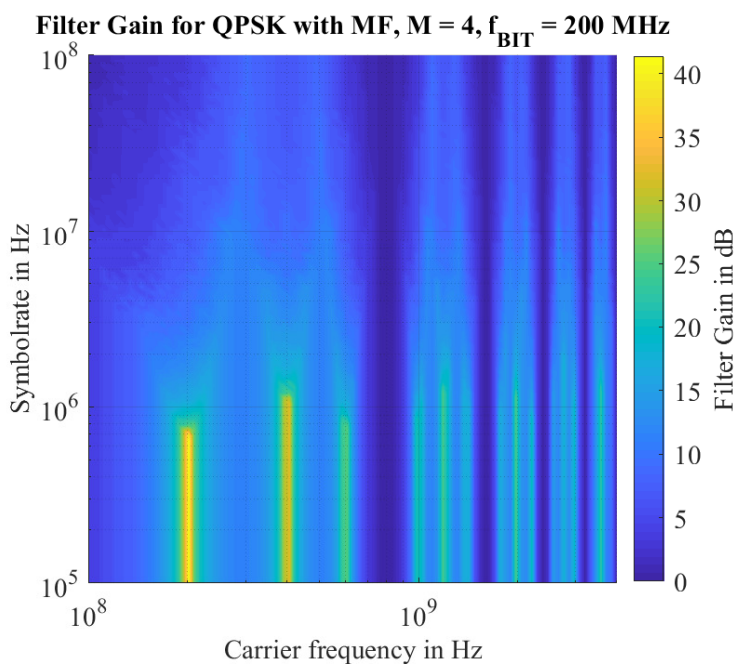

(c) QPSK

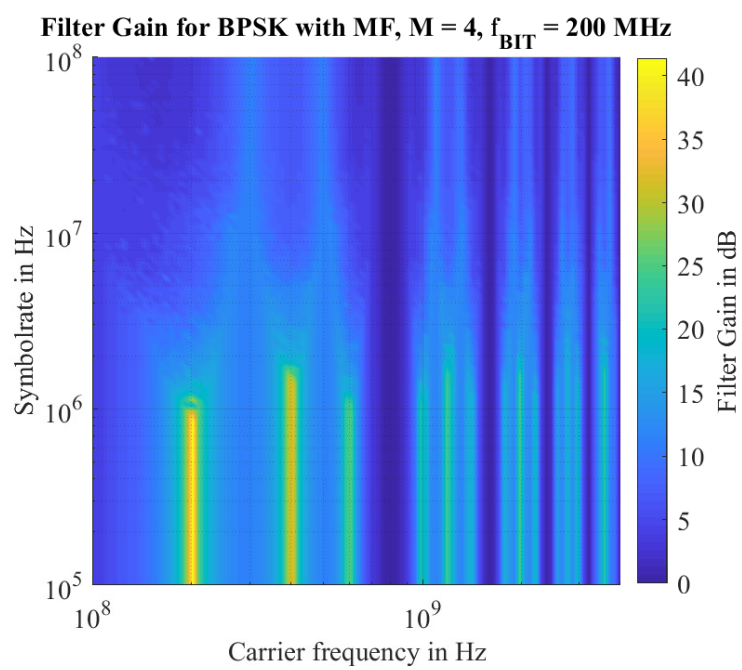

(b) BPSK

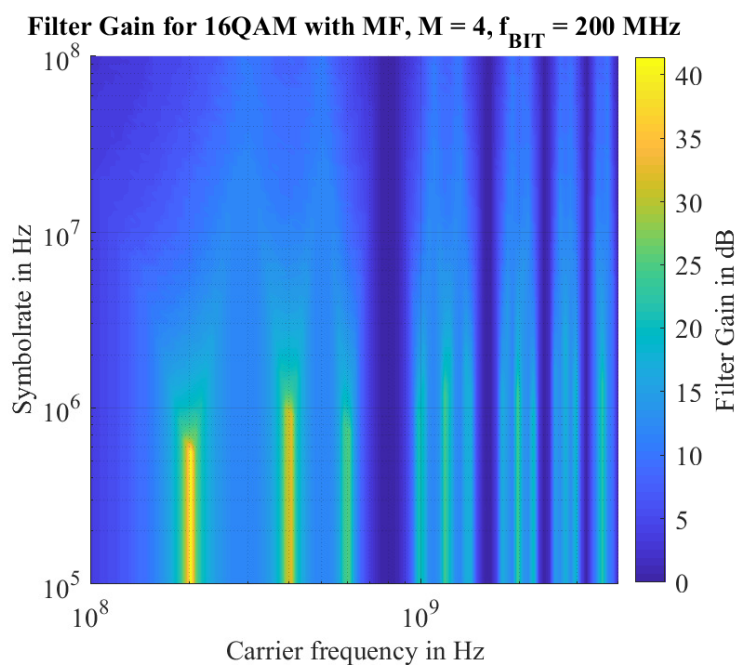

(d) 16-QAM

Fig. 11. The Filter Gain at BER $=0.1 \%$ for CW, BPSK, QPSK and 16-QAM in function of $f_{c}$ and $f_{\mathrm{SYM}}$ for an $f_{\mathrm{BIT}}=200 \mathrm{MHz}$ and $M=4$.

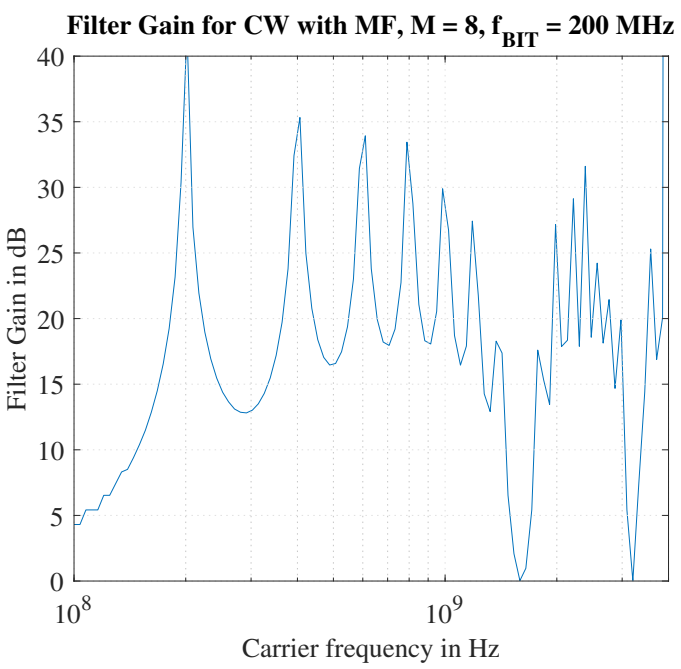

(a) $\mathrm{CW}$

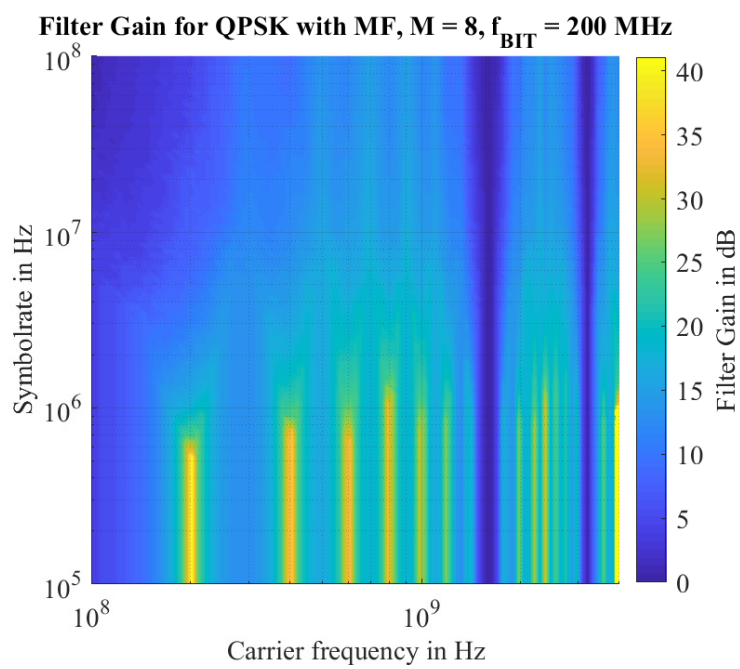

(b) QPSK

Fig. 12. The Filter Gain at $\mathrm{BER}=0.1 \%$ for $\mathrm{CW}$ and QPSK in function of $f_{c}$ and $f_{\mathrm{SYM}}$ for an $f_{\mathrm{BIT}}=200 \mathrm{MHz}$ and $M=8$. 
[11] J. Lannoo, A. Degraeve, D. Vanoost, J. Boydens, and D. Pissoort, "Effectiveness of inversion diversity to cope with EMI within a twochannel redundant system," in 2018 IEEE International Symposium on Electromagnetic Compatibility and 2018 IEEE Asia-Pacific Symposium on Electromagnetic Compatibility (EMC/APEMC), May 2018, pp. 216220.

[12] A. Degraeve and D. Pissoort, "Study of the effectiveness of spatially EM-diverse redundant systems under plane-wave illumination," in 2016 Asia-Pacific International Symposium on Electromagnetic Compatibility (APEMC), vol. 01, May 2016, pp. 211-213.

[13] — "Study of the effectiveness of spatially EM-diverse redundant systems under reverberation room conditions," in 2016 IEEE International Symposium on Electromagnetic Compatibility (EMC), July 2016, pp. 374-378.

[14] J. Lannoo, J. Van Waes, A. Degraeve, D. Vanoost, J. Boydens, and D. Pissoort, "Effectiveness of time diversity to obtain EMI-diverse redundant systems," in 2018 International Symposium on Electromagnetic Compatibility (EMC EUROPE), Aug 2018, pp. 288-292.

[15] H. Hsu, Schaum's Outline of Analog and Digital Communications, ser Schaum's Outline Series. McGraw-Hill Education, 2012. [Online]. Available: https://books.google.be/books?id=Eq6oLxVNkLQC

[16] Huang Dongjie and Shao Wenchang, "A digital realization technique of optimum receiving matched filter," in China., 1991 International Conference on Circuits and Systems, 1991, pp. 181-184 vol.1.

[17] C. Ruan, J. Hua, Z. Zheng, Y. Wu, and L. Meng, "A study of different matched filters in digital down converter," in 2012 International Conference on Systems and Informatics (ICSAI2012), 2012, pp. 20592063.

[18] V. . Kaasila and A. Mammela, "Bit error probability of a matched filter in a Rayleigh fading multipath channel," IEEE Transactions on Communications, vol. 42, no. 234, pp. 826-828, 1994.

[19] J. Lannoo, J. V. Waes, D. Vanoost, J. Boydens, and D. Pissoort, "The effectiveness of a matched filter to cope with harsh continuous wave EMI," in 2019 IEEE International Symposium on Electromagnetic Compatibility, Signal Power Integrity (EMC+SIPI), July 2019, pp. 3539.

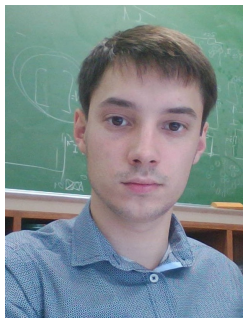

Jonas Lannoo Jonas Lannoo was born in Brugge, Belgium, on August 2, 1993. He received the M.S. degree in Engineering Technology, Electronics, from KU Leuven, Technology Campus Ostend, Belgium, in 2016. He is currently working towards the Ph.D. degree in electrical engineering. Since 2016, he has been a Research Assistant at the M-Group research group, KU Leuven Bruges Campus, Bruges, Belgium, which is a research group focusing on global dependability of mechatronic systems. His research interests are electromagnetic compatibility, functional safety and electromagnetic interference on a system level, ageing of electrical components and systems, but also power quality and vibration analysis in electrical machines.

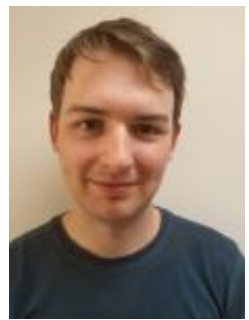

Tim Claeys Tim Claeys was born in 1990. He received the M.S. industrial engineering sciences, option electronics, from the University College Katholieke hogeschool Sint-Lieven Gent (KAHO Sint-Lieven), Ghent, Belgium, in 2013. He received the Ph.D. Degree from KU Leuven, Leuven, Belgium, in 2018. Since 2018, he is a Post-doctoral researcher at the M-Group research group (lab FMEC), KU Leuven Bruges Campus, which is a laboratory focusing on global reliability of electronic systems and Electromagnetic Compatibility. His research interests are Near-field scanning, the development of characterization methods for shielding materials and gaskets, EMI resilience of wireless protocols and global reliability of electronic systems.

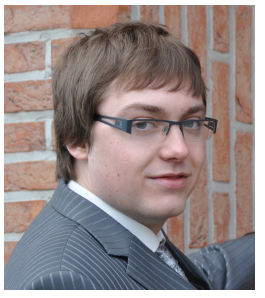

Dries Vanoost Dries Vanoost was born in 1988. He received the M.S. degree from KU Leuven Technology campus Ostend, Ostend, Belgium, in 2010, and the $\mathrm{PhD}$ degree from KU Leuven, Leuven, Belgium, in 2015, both in electrical engineering. Since January 2016, he has been a Postdoctoral Researcher at the M-Group research group, KU Leuven Bruges Campus, which is a laboratory focusing on global reliability of mechatronic systems and electromagnetic compatibility. His research interests include interconnected mechatronic systems, electromagnetic modelling, power electronics, and power quality.

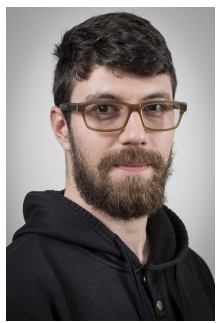

Jonas Van Waes J. Van Waes was born in Ghent, Belgium in 1991. He received the B.S. and M.S. degrees in Electronics and ICT in respectivily 2014 and 2016. He is currently persuing a $\mathrm{PhD}$ degree in Engineering Technology at the KU Leuven campus in Bruges, Belgium. Meanwhile, he is working as a Research Assistant in the same institute, being a part of the Mechatronics research group, which is a research group focusing on global dependability of mechatronic systems. His research is to limit the influence of electromagnetic interference in safetycritical embedded systems residing in harsh electromagnetic environments. He mainly focuses on the protection of (crucial) data communication and the occurrences of false negatives.

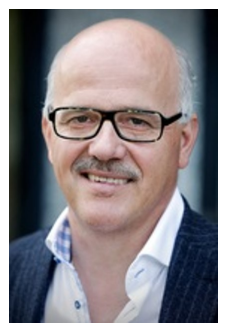

Jeroen Boydens Jeroen Boydens is a professor at $\mathrm{M}$-Group research group at $\mathrm{KU}$ Leuven, Campus Bruges in Belgium where he leads a team of doctoral researchers in the field of embedded software. He received his M.S. in Electromechanical Engineering at KIH (Belgium) in 1988 and his M.S. in Computer Science from VUB (Brussels Belgium) in 1989 In 2008 he received his Ph.D. from KU Leuven in the field of adding constructs to object-oriented programming languages. From September 2011 to September 2017, he was an Assistant Professor and since October 2017an Associate Professor, both at KU Leuven, Belgium.In 2007 he started his research group on embedded software engineering where he led a number of projects in close cooperation with industry. Techniques such as the detection of disturbances due to EMI in software and techniques to recover are active research fields. Since August 2017 he is program director for Electronics-ICT in Faculty of Engineering, KU Leuven. Since October 2017 he is a visiting professor at Université de Limoges(FR), for a course on embedded software development. He's an IEEE-member since 2015.

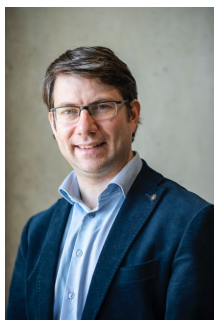

Davy Pissoort Davy Pissoort (M'09-SM'13) was born in 1978 . He received the M.S and Ph.D. degrees in electrical engineering from Ghent University, Ghent, Belgium, in 2001 and 2005, respectively. From October 2005 to October 2006, he was a Postdoctoral Researcher at Ghent University. From November 2006 to July 2009, he was a Research Engineer in the Eesof-EDA Department, Agilent Technolgies, Belgium. From August 2009 to September 2016, he was an Assistant Professor and since October 2016 an Associate Professor, both at KU Leuven, Belgium. His current research interests include the development of fast and efficient electromagnetic modeling methods for EMC, SI, and PI, the development of characterization methods for shielding materials and gaskets, EMI Risk Management as well as the analysis and testing of the mechanical and thermal reliability of electronic modules. Dr. Pissoort is a Senior Member of the IEEE EMC Society, IEEE EMC Society Board Member and member of the International Steering Committee of EMC Europe. 